\title{
Threshold Mass Numerologies and Resonances \\ in Boson and Baryon Systems.
}

\author{
S. F. TUAN \\ Department of Physics, Brown University - Providence, R.I. \\ (Nuovo Cimento, 23, $448(1962))$
}

The last but one paragraph of the note added in proof of $p .452$ should correctly read:

"Professor Frazer (private communication) has questioned the "strength" of $\zeta$ to saturate the unitarity limit needed for an explanation of $\mathcal{N}^{* *}$ along the lines of the Frazer-Ball threshold model. Perhaps $\zeta$ is "induced" by some other dynamical effects such as the isobar mechanism based on correlations amongst the three body system $\pi+\pi+N^{\prime}$, 